\title{
Effects of sevoflurane on rats with ischemic brain injury and the role of the TREK-1 channel
}

\author{
LIXIAO PAN $^{1}$, FENGYUN YANG ${ }^{1}$, CAIXIA LU $^{2}$, CHANGXIN JIA ${ }^{1}$, \\ QING WANG ${ }^{3}$ and KEXUE ZENG ${ }^{4}$
}

\author{
Departments of ${ }^{1}$ Anesthesiology, ${ }^{2}$ Obstetrics and ${ }^{3}$ Endocrinology, The Affiliated Hospital of \\ Qingdao University, Qingdao, Shandong 266000; ${ }^{4}$ Department of Massage, Guangdong Second \\ Traditional Chinese Medicine Hospital, Guangzhou, Guangdong 510095, P.R. China
}

Received May 18, 2017; Accepted July 25, 2017

DOI: $10.3892 /$ etm.2017.4906

\begin{abstract}
The purpose of this investigation was to determine the effects of sevoflurane on rats with ischemic brain injury and to determine the potential role of the TREK-1 channel in this process. Normal rats were randomly divided into three groups: Sham operation, sevoflurane anesthesia or chloral hydrate anesthesia group, an additional group of TREK-1 knockout rats were also studied. Semi-quantitative PCR and western blot analysis confirmed the lack of TREK-1 expression in the brain of TREK-1 knockout rats. The thread-tie method was used to establish middle cerebral artery occlusion (MCAO) model to induce cerebral ischemic brain injury. All rates were treated for 4 days prior to ischemia (for $2 \mathrm{~h}$ ) followed by a $24 \mathrm{~h}$ reperfusion period. Physiological indexes of rats in each group both prior to and after surgery showed no statistical difference $(\mathrm{P}>0.05)$. Neurological function was scored both before (no statistical difference) and after surgery where it was found to be significantly better (lower score) in the sevoflurane anesthesia group than in chloral hydrate anesthesia and TREK-1 knockout groups $(\mathrm{P}<0.01)$. The area of cerebral infarction was measured by triphenyl tetrazolium chloride staining and terminal deoxynucleotidyl transferase dUTP nick end labeling (TUNEL) assay to detect the apoptosis of brain cells. TTC staining showed different degrees of cerebral infarction in the various groups; the area of cerebral infarction in sevoflurane anesthesia group was significantly lower than that in chloral hydrate anesthesia and TREK-1 knockout groups $(\mathrm{P}<0.01)$. TUNEL assay showed that the number of TUNEL-positive cells was significantly lower in sevoflurane anesthesia group than in TREK-1 knockout and chloral hydrate anesthesia groups $(\mathrm{P}<0.01)$. In conclusion, results from this investigation
\end{abstract}

Correspondence to: Dr Kexue Zeng, Department of Massage, Guangdong Second Traditional Chinese Medicine Hospital, 60 Hengfu Road, Yuexiu, Guangzhou, Guangdong 510095, P.R. China E-mail: kexue_zeng@163.com

Key words: cerebral ischemic brain injury, sevoflurane, middle cerebral artery occlusion, model, rat showed that sevoflurane can protect the nerve function of rats with cerebral ischemic brain injury possibly by affecting the expression of proteins involved in the TREK-1 signaling pathway.

\section{Introduction}

Cerebrovascular accident (also known as a stroke), is the second ranked disease to cause human mortality. Stroke often leads to marked disability, that not only affects the patients' life quality but also creates an economic burden to both the patients' family and society (1-3). Stroke is clinically divided into either ischemic or hemorrhagic stroke types, of which ischemic stroke accounts for about $80 \%$ of all cases (4-6). Causality of ischemic stroke is due to an insufficient blood and oxygen supply to brain caused by various etiologies, resulting in brain tissue necrosis. Blood flow reperfusion after cerebral ischemia often cannot restore normal brain function, but can further worsen tissue damage and dysfunction; this phenomenon is termed ischemia-reperfusion injury (7-9). Sevoflurane, a volatile anesthetic, is commonly used in clinical practice due to its efficacy and safety profile. Studies have shown that sevoflurane pretreatment has a neuroprotective effect on mice subjected to cerebral ischemia. The acute onset of cerebral ischemia can activate the TREK-1 channel to protect the tissue (10), however, the possible relationship between and mechanism of action of sevoflurane and the TREK-1 channel has not yet been delineated. This investigation determined whether the neuroprotective effect of sevoflurane on induced cerebral ischemia in rodents [middle cerebral artery occlusion (MCAO) model] is due to the activation of the TREK-1 channel and if so to determine its induction mechanism.

\section{Materials and methods}

Male Sprague-Dawley (SD) rats (8 weeks old, 250-300 g) were provided by Guangdong Medical Laboratory Animal Center (Foshan, China) [Certificate of Quality No: SCXK (Guangdong) 2015-0019]. TREK-1 knockout rats (8 weeks, 250-300 g) were purchased from Cyagen Biosciences Inc., (Guangzhou, China). All rats were kept in a quiet feeding environment $\left(25^{\circ} \mathrm{C}\right)$ with strict compliance with circadian 
rhythm for 1 week prior to initiation of the experiments and allowed ad libitum food and water. A total of 36 SD rats were randomly divided into either the sham operation, sevoflurane (Baxter International Inc., Deerfield, IL, USA), anesthesia or chloral hydrate (Aladdin Bio-Chem Technology Co., Ltd., Shanghai, China) anesthesia group, 12 rats in each group. A fourth group of 12, TREK-1 knockout rats were also utilized. All experimental animals except for the chloral hydrate anesthesia group were subjected to pretreatment for 4 days: The sevoflurane anesthesia and 12 TREK-1 knockout inhaled sevoflurane (sevoflurane/air, 1:2) for 30 min every day using a respiratory anesthesia machine; the sham operation group could breathe freely; and the chloral hydrate anesthesia group were treated with chloral hydrate $(4 \%, 1 \mathrm{ml} / 100 \mathrm{~g})$ once a day. This study was approved by the Animal Ethics Committee of Qingdao University Animal Center.

The MCAO model was established as follows. All rats except for the chloral hydrate group (intraperitoneally injected $4 \%$ chloral hydrate anesthesia) were subjected to sevoflurane anesthesia. Animals were fixed to the operating table, shaved and the common carotid artery was isolated; the internal and external carotid artery were bluntly separated along the left common carotid artery. The base of external and common carotid artery were then ligated. After clamping the distal end of internal carotid artery with artery clips, syringe needle was used to make an incision at the proximal end of the internal carotid artery to insert a nylon thread $(0.3 \mathrm{~mm}$ diameter $)$ with a depth of $18.0 \pm 2.0 \mathrm{~mm}$ to reach the middle of the middle cerebral artery, to completely block arterial blood supply. Internal carotid artery was then ligated. The surgical wound was closed and the ligation on the middle cerebral artery was left outside the skin; rats were placed on a constant temperature blanket until they were resuscitated. After $2 \mathrm{~h}$ of ischemia, the nylon thread was removed and reperfusion was allowed for $24 \mathrm{~h}$. The sham rat operation group was subjected to the same surgical procedure with exception to the nylon thread insertion.

Pretreatment physiological indices (plasma $\mathrm{pH}, \mathrm{PaCO}_{2}$, $\mathrm{PaO}_{2}$ and body temperature) were monitored using a physiological signal recorder (Powerlab group, Nuremberg, Germany) to rule out the effects of hypoxia. Rats in sham operation, sevoflurane anesthesia and knockout group were rapidly resuscitated after sevoflurane inhalation for 20 min using a breather box and then treated with sevoflurane for another 10 min using a nasal mask. During this $10 \mathrm{~min}$, left femoral artery catheterization was performed and blood samples were collected for blood gas analysis $10 \mathrm{~min}$ later. Rectal temperature was also measured. After intraperitoneal injection of chloral hydrate, samples from rats in chloral hydrate anesthesia group at the same time points using the same methods for blood gas analysis and rectal temperature was also measured. Physiological indexes (changes in animal blood gas, pressure and body temperature) were closely monitored during surgery. Rats were fixed in supine position for surgery. Incision was made on left groin to separate femoral artery and heparin-treated pressure transducer was then inserted. The blood pressure and arterial blood gas were monitored at 20 min before and after the insertion of nylon thread and $20 \mathrm{~min}$ after the removal. Rectal temperature of the rats was also monitored during the surgery.

Scoring of neurological function was performed at 2, 8 and $24 \mathrm{~h}$ after cerebral ischemia-reperfusion according
Table I. Primer sequences.

Sequences

$\begin{array}{ll}\text { TREK-1 } & \text { F: 5'-ATCCAGAGACAAGACATGTAC-3' } \\ & \text { R: 5'-TTCAGATGTTCTAAGCCTACGG-3' } \\ \text { GAPDH } & \text { F: 5'-GATGATTGGCATGGCTTT-3' } \\ & \text { R: 5'-CACCTTCCGTTCCAGTTT-3' }\end{array}$

F, forward; R, reverse; GAPDH, glyceraldehyde 3-phosphate dehydrogenase.

to Zea-Longa scoring criteria. 0, no neurological deficit symptoms with normal activity; 1, contralateral forepaws of the rats cannot stretch freely (mild neurological deficits); 2, show contralateral rotation during walking (moderate neurological deficit); 3, show contralateral falling during walking (severe neurological deficits); 4, fail to walk spontaneously and show loss of consciousness. The total scores of neurological functions of all rats were analyzed statistically.

After reperfusion for $24 \mathrm{~h}$ and the completion of neurological function scoring, rats were sacrificed and the brain was prepared into $3 \mathrm{~mm}$ sections for triphenyl tetrazolium chloride (TCC) (Sigma-Aldrich, St. Louis, MO, USA) staining. Brain tissue sections were incubated with $2 \%$ TTC solution at $37^{\circ} \mathrm{C}$ in the dark for $5 \mathrm{~min}$; the sections were then fixed in $4 \%$ paraformaldehyde. Tissue section with living tissue have a bright red color, while necrotic tissue was pale in appearance consistent with tissue necrosis. After fixation for $24 \mathrm{~h}$, digital images were analyzed by image processing software to calculate the percentage area of cerebral infarction tissue.

Determination of brain cell apoptosis was performed using a terminal deoxynucleotidyl transferase dUTP nick end labeling (TUNEL) apoptosis detection kit (Invitrogen Life Technologies, Carlsbad, CA, USA) according to the manufactures protocol. After dewaxing and hydration, brain tissue sections were treated with fresh prepared $3 \%$ hydrogen peroxide for $10 \mathrm{~min}$, followed by washing 3 times with phosphate-buffered saline (PBS). Tissue sections were digested by proteinase $\mathrm{K}$ in a wet box at $37^{\circ} \mathrm{C}$ for $10 \mathrm{~min}$, followed by washing twice with PBS. TdT $(40 \mu \mathrm{l})$ and DIG-d-UTP mixture was then added and incubated at $4^{\circ} \mathrm{C}$ for $2 \mathrm{~h}$, followed by washing with PBS for three times. Forty microliters of blocking solution was added and allowed to stand at room temperature for $30 \mathrm{~min}$. Biotinylated anti-digoxin antibody (1:100) was then added and incubated in a wet box at $37^{\circ} \mathrm{C}$ for $40 \mathrm{~min}$, followed by washing 3 times with PBS. SABC-FITC secondary antibody (1:100) was then added and incubated in a wet box at $37^{\circ} \mathrm{C}$ for $40 \mathrm{~min}$, followed by washing 3 times with PBS. The slides were sealed with anti-fluorescence quenching liquid and brain tissue sections were observed under a fluorescence microscope and digital images were analyzed (cells with yellow-green fluorescence were consider positive apoptotic cells).

The expression of TREK-1 gene at mRNA and protein levels was determined from tissue samples. RNA was extracted from brain tissue with TRIzol (Invitrogen Life Technologies) 
Table II. Effects of different anesthesia models on physiologic indexes during pretreatment.

\begin{tabular}{lcccc}
\hline Physiological indexes & $\mathrm{pH}$ & $\mathrm{PaCO}_{2}(\mathrm{mmHg})$ & $\mathrm{PaO}_{2}(\mathrm{mmHg})$ & Rectal temperature $\left({ }^{\circ} \mathrm{C}\right)$ \\
\hline Chloral hydrate anesthesia group & $7.38 \pm 0.06$ & $38.9 \pm 3.7$ & $95.3 \pm 5.5$ & $37.2 \pm 0.2$ \\
Sevoflurane anesthesia group & $7.32 \pm 0.03$ & $39.7 \pm 3.1$ & $98.6 \pm 3.7$ & $36.9 \pm 0.3$ \\
P-value & $>0.05$ & $>0.05$ & $>0.05$ & $>0.05$ \\
\hline
\end{tabular}

Table III. Effects of different anesthesia models on physiologic indexes during surgery.

\begin{tabular}{lcccc}
\hline Physiological indexes & $\mathrm{pH}$ & $\mathrm{PaCO}_{2}(\mathrm{mmHg})$ & $\mathrm{PaO}_{2}(\mathrm{mmHg})$ & Rectal temperature $\left({ }^{\circ} \mathrm{C}\right)$ \\
\hline Chloral hydrate anesthesia group & $7.27 \pm 0.04$ & $41.2 \pm 2.8$ & $98.3 \pm 2.6$ & $36.8 \pm 0.4$ \\
Sevoflurane anesthesia group & $7.31 \pm 0.08$ & $42.6 \pm 3.5$ & $97.9 \pm 3.8$ & $37.0 \pm 0.3$ \\
P-value & $>0.05$ & $>0.05$ & $>0.05$ & $>0.05$ \\
\hline
\end{tabular}

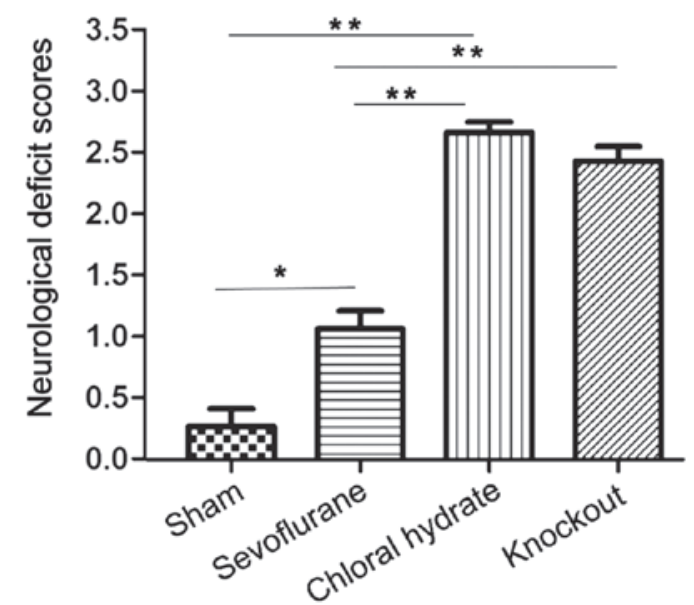

Figure 1. Neurological deficit reflected by neurological function score. The score of sevoflurane anesthesia, chloral hydrate anesthesia and knockout group was significantly higher than that of sham operation group; score was significantly lower in sevoflurane anesthesia than in chloral hydrate anesthesia and knockout group. No significant difference was found between chloral hydrate anesthesia and knockout group. Compared with sham operation group, ${ }^{*} \mathrm{P}<0.05$; compared with sham operation group, ${ }^{* *} \mathrm{P}<0.01$; Compared with sevoflurane anesthesia group, $\mathrm{P}<0.01$.

(RNA integrity was verified by agarose gel electrophoresis showing bands of 28, 18 and 5S RNA were clear and the brightness of the $28 \mathrm{~S}$ was about twice that of $18 \mathrm{~S}$ RNA). The RNA was subjected to reverse transcription, expression of TREK-1 mRNA was detected by semi-quantitative PCR (qPCR) using GAPDH as endogenous control. The reaction conditions were: $95^{\circ} \mathrm{C}$ for $5 \mathrm{~min}$, followed by 35 cycles of $95^{\circ} \mathrm{C}$ for $30 \mathrm{sec}, 64^{\circ} \mathrm{C}$ for $25 \mathrm{sec}$ and $72^{\circ} \mathrm{C}$ for $30 \mathrm{sec}$ and $72^{\circ} \mathrm{C}$ for $5 \mathrm{~min}$. TREK-1 primers (Table I) were synthesized by Tiangen Biotech Co., Ltd. (Beijing, China). After reaction, agarose gel electrophoresis was performed and the bands resolved using an UV imaging system. Brain tissue samples, that had been stored in liquid nitrogen, underwent western blot analysis. Samples were thawed and then lysed using RIPA lysate $(100 \mathrm{mg} / 1 \mathrm{ml})$. After centrifugation for $5 \mathrm{~min}$ at $850 \mathrm{x} \mathrm{g}$, the supernatant was collected and total protein quantitated using a BCA protein quantification kit. Protein samples were subjected to SDS polyacrylamide gel electrophoresis and then transferred to PVDF membrane under $100 \mathrm{~V}$ for $90 \mathrm{~min}$. After blocking for $2 \mathrm{~h}$, the membrane was incubated with rabbit antiTREK-1 antibody (Cell Signaling Technology, Inc., Danvers, MA, USA) overnight at $4^{\circ} \mathrm{C}$, followed by washing 3 times for 5 min with Tris-buffered saline Tween-20 TBST. The membrane was incubated with secondary antibody for $2 \mathrm{~h}$, followed by washing 3 times with TBST. ECL luminescent liquid (Invitrogen Life Technologies), (mixture of liquid A and liquid $\mathrm{B}$ with 1:1 ratio) was added and the membrane incubated until the bands could be resolved by fluorescence intensity. After color development, the blots were scanned and gray values of the bands were analyzed using ImageJ software.

Statistical analysis. All the data were expressed as mean \pm standard deviation and processed by SPSS 19.0 software (SPSS Inc., Chicago, IL, USA). Comparisons between 2 groups were performed using t-test and comparisons among multiple groups were performed using variance analysis. Homogeneity test of variance was performed. If the variance was homogeneous, the comparisons between two groups were performed using Bonferroni correction method. If the variance was not homogeneous, Welch method was used. Multiple comparisons were performed using Dunnett's T3 method. $\mathrm{P}<0.05$ was considered to indicate a statistically significant difference.

\section{Results}

Physiological indexes from the rat were monitored to rule out the effects of different anesthesia methods during pretreatment and surgery. Blood gas and body temperature of the SD rats from rats in each group were not statistically $(\mathrm{P}>0.05)$ different (Tables II and III).

The neurological function of SD rats in each group were all 0 points before surgery. After operation, neurological function of rats in sham operation group, sevoflurane anesthesia, chloral hydrate anesthesia and knockout group were scored (Fig. 1). The neurological function score was significantly higher in sevoflurane anesthesia, chloral hydrate anesthesia 
A

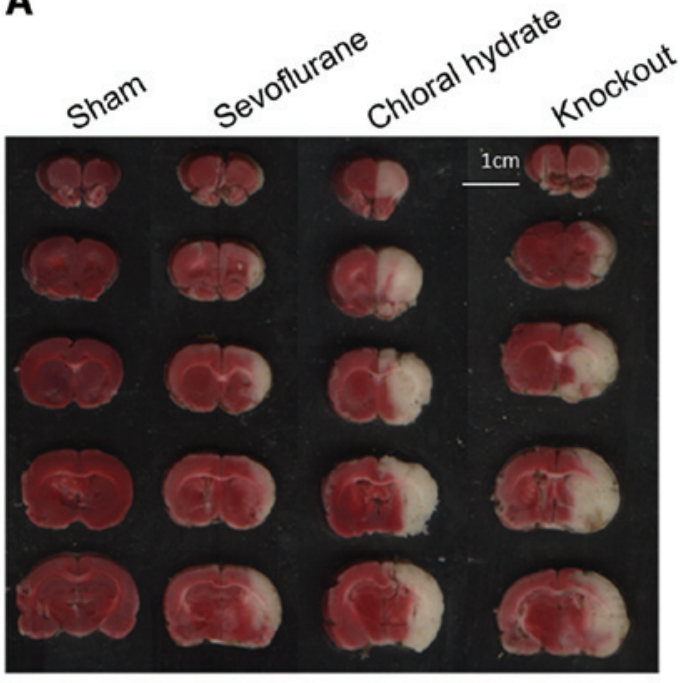

B

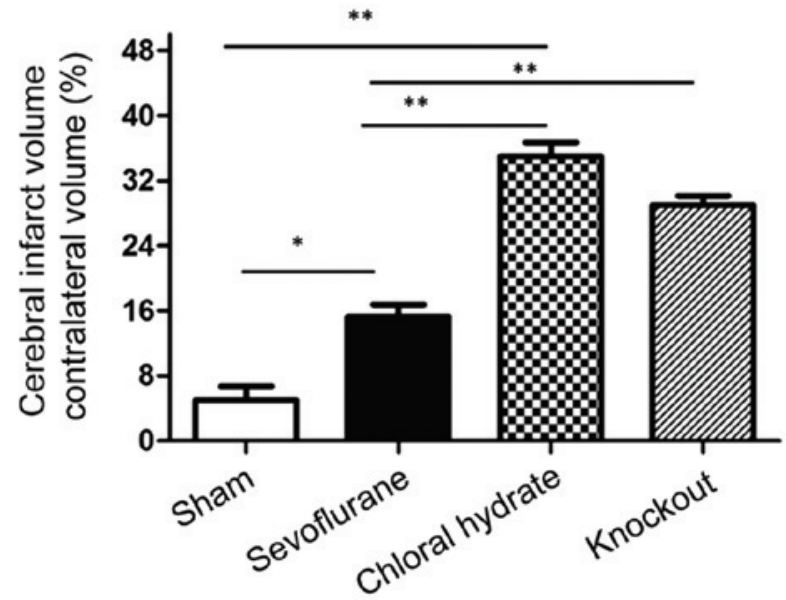

Figure 2. TTC staining results of brain tissue sections from each group. (A) Representative TTC staining results. Scale bar, $1 \mathrm{~cm}$; (B) summary of cerebral infarction area. Cerebral infarction area in sevoflurane anesthesia, chloral hydrate anesthesia and knockout group was significantly larger than that in sham operation group, while the infarction area of sevoflurane anesthesia was significantly smaller than that of chloral hydrate anesthesia and knockout group. No significant difference was found between chloral hydrate anesthesia and knockout group. Compared with sham operation group, ${ }^{*} \mathrm{P}<0.05$; compared with sham operation group, ${ }^{* *} \mathrm{P}<0.01$; Compared with sevoflurane anesthesia group, $\mathrm{P}<0.01$. TTC, triphenyl tetrazolium chloride.
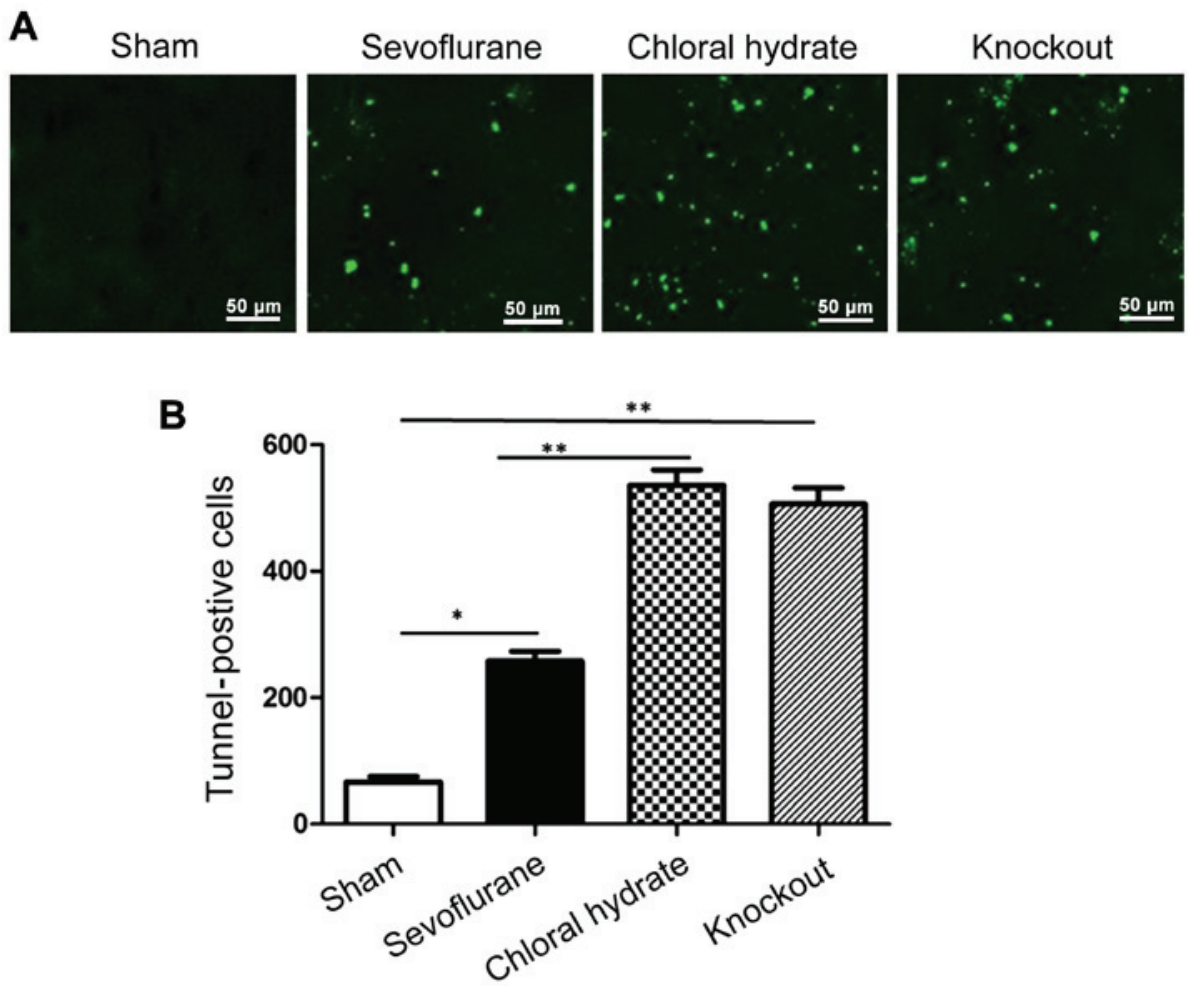

Figure 3. Apoptotic cells detected by TUNEL staining. (A) Representative results of the TUNEL staining; (B) summary of brain cell apoptosis. Compared with sham operation group, the number of TUNEL-positive cells was significantly increased in sevoflurane anesthesia, chloral hydrate anesthesia and knockout group. The number of TUNEL-positive cells in sevoflurane anesthesia group was significantly lower than that in chloral hydrate anesthesia and knockout group. No significant difference between chloral hydrate anesthesia and knockout group. Compared with sham operation group, ${ }^{*} \mathrm{P}<0.05 ;$ compared with sham operation group, ${ }^{* *} \mathrm{P}<0.01$; Compared with sevoflurane anesthesia group, $\mathrm{P}<0.01$.

and knockout group than in sham operation group $(\mathrm{P}<0.05$ or $\mathrm{P}<0.01$ ), while the neurological function score of sevoflurane anesthesia was significantly lower than that of chloral hydrate anesthesia and knockout group $(\mathrm{P}<0.01)$. No significant difference was found between chloral hydrate anesthesia and knockout group $(\mathrm{P}>0.05)$.

The cerebral infarction area was determined using TTC staining. Pretreatment of sevoflurane and chloral hydrate 


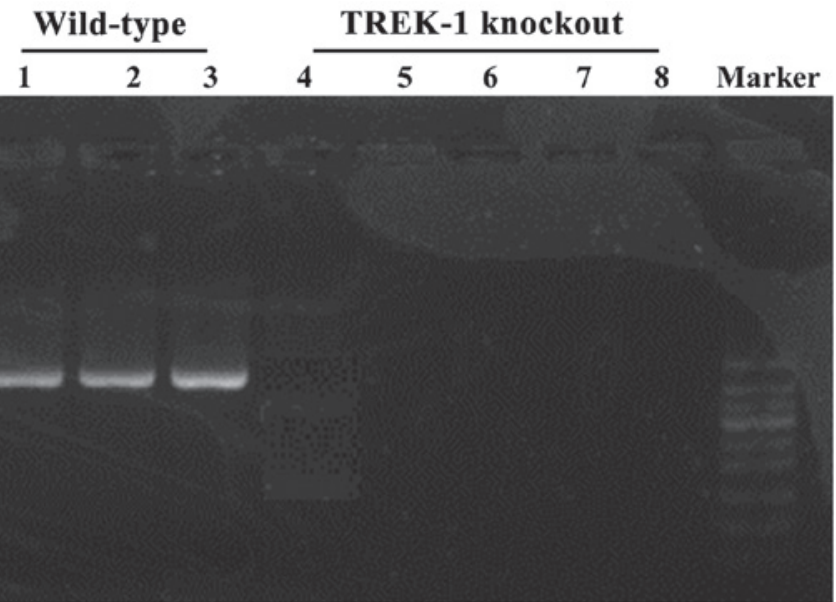

Figure 4. Expression level of TREK-1 mRNA detected by semi-qPCR Lanes 1-3, wild-type rats. Lanes 4-8, TREK-1 knockout mice. Results showed that, compared with wild-type rat, TREK-1 knockout rats did not express TREK-1.
A

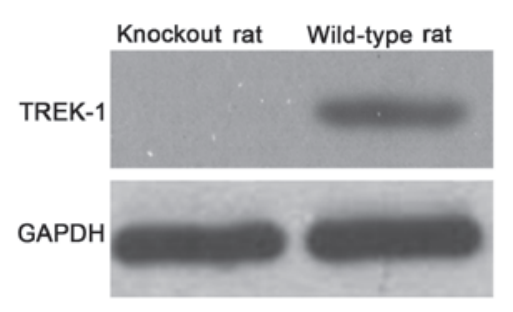

B

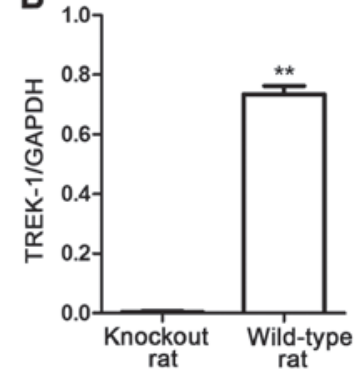

Figure 5. Expression of TREK-1 protein detected by western blot analysis. (A) Results of western blot analysis; (B) relative protein expression level. The results showed that, compared with wild-type rats, TREK-1 knockout rat did not express TREK-1 protein. Compared with TREK-1 knockout rat, ${ }^{* *} \mathrm{P}<0.01$. GAPDH, glyceraldehyde 3-phosphate dehydrogenase.

anesthesia was initiated 4 days before surgery. Occlusion of the middle cerebral artery was performed for $2 \mathrm{~h}$ followed by reperfusion for $24 \mathrm{~h}$. Rats were sacrificed and brain tissue was subjected to TTC staining (Fig. 2). The cerebral infarction area was significantly larger in sevoflurane anesthesia group, chloral hydrate anesthesia group and knockout group than in sham operation group $(\mathrm{P}<0.05$ or $\mathrm{P}<0.01)$, while the infarction area of sevoflurane anesthesia group was significantly smaller than that of chloral hydrate anesthesia group and knockout group $(\mathrm{P}<0.01)$. No significant difference was found between chloral hydrate anesthesia group and knockout group ( $\mathrm{P}>0.05)$.

The number of apoptotic brain cells between groups was determined using TUNEL staining (Fig. 3). After reperfusion for $24 \mathrm{~h}$, no TUNEL-positive cells were observed in the cortex of brain tissue derived from the rats in sham operation group. Compared with sham operation group, the number of TUNEL-positive cells was significantly increased in sevoflurane anesthesia, chloral hydrate anesthesia and knockout group $(\mathrm{P}<0.01$ or $\mathrm{P}<0.05)$. The number of TUNEL-positive cells in sevoflurane anesthesia was significantly lower than that in chloral hydrate anesthesia and knockout group $(\mathrm{P}<0.01)$, but there was no significant difference between chloral hydrate anesthesia and knockout group $(\mathrm{P}>0.05)$.
The expression of TREK-1 in TREK-1 knockout rat was detected by semi-qPCR and western blot analysis (Figs. 4 and 5). TREK-1 mRNA could not be detected in TREK-1 knockout rats by semi-qPCR (Fig. 4), in addition, compared with wild-type rat, TREK-1 protein could not be detected by western blot analysis $(\mathrm{P}<0.01)$ (Fig. 5), indicating that TREK-1 knockout rat model was established.

\section{Discussion}

Stroke is a major cause of both human health mortality (one of the three major causes of human fatality along with cardiovascular disease and cancer) and morbidity $(11,12)$. In developed countries, ischemic cerebrovascular disease accounts for $85 \%$ of all stroke cases. Ischemic stroke is caused by the embolization or thrombosis in brain blood vessels, especially in cerebral arteries, which can interrupt brain blood supply, resulting in disorders of energy metabolism, ionic homeostasis imbalance and the production of free radical and excitatory neurotoxicity (13). TREK-1 channel, which is a novel subtype of two-pore-domain potassium channels, can be activated by mechanical stretch and polyunsaturated fatty acids. Numerous studies have reported that TREK-1 (mainly expressed in brain and spinal cord) channel plays a neuroprotective effect in the process of cerebral ischemic injury $(14,15)$. Treatment with TREK-1 channel agonists can effectively reduce brain damage in animal models of cerebral ischemia and epilepsy, while brain damage cannot be reduced in TREK-1 knockout rats (16). The activated TREK-1 channel can play a neuroprotective effect by regulating the dynamic balance of water and ions and removing excitotoxin substances and oxygen free radicals $(17,18)$. Sevoflurane, as a commonly used anesthetic, is frequently used in animal experiments and clinical practice due to its effectiveness and safety profile. Studies have shown that sevoflurane can activate TREK-1 pathway through an indirect pathway at the cellular level, thereby preventing cell damage caused by ischemia and hypoxia $(19,20)$.

In this study, the neuroprotective effect of sevoflurane on rats with cerebral ischemia and the relationship with TREK-1 channel was investigated. Semi-qPCR and western blot analysis were used to confirm that TREK-1 knocked out at both mRNA and protein levels. The amount of the cerebral infarction area was calculated and the number of apoptotic cells in brain tissue was determined. Results showed that sevoflurane had a stronger neuroprotective effect on rats with ischemic brain injury compared with chloral hydrate $(\mathrm{P}<0.01)$. Sevoflurane showed no neuroprotective effect on TREK-1 knockout rats with ischemic brain injury $(\mathrm{P}>0.05)$ and the cerebral infarction area and the number of apoptotic cells showed no significant difference from those of chloral hydrate anesthesia group, indicating that the neuroprotective effect of sevoflurane is possibly achieved through TREK-1 channel. To rule out the effects of sevoflurane and chloral hydrate on physiological indexes during pretreatment and surgery, physiological indexes of rats in each group were monitored; results showed no significant effect on the physiological indexes of rats in each group, indicating that the neuroprotective effect of sevoflurane on ischemic brain injury is not achieved by affecting the physiological indexes. These results suggest that 
the neuroprotective effect of sevoflurane on ischemic brain injury in rats may be achieved by affecting the expression of related proteins involved in TREK-1 channel pathway; however, the precise mechanism at a molecular level is not yet elucidated.

In conclusion, sevoflurane has a neuroprotective effect on rats with cerebral ischemia, which can significantly reduce cerebral infarction area and number of apoptotic cells and the neuroprotective effect of sevoflurane is possibly achieved through its regulation on the TREK-1 channel.

\section{References}

1. Park JS, Hwang NK, Oh DH and Chang MY: Effect of head lift exercise on kinematic motion of the hyolaryngeal complex and aspiration in patients with dysphagic stroke. J Oral Rehabil 44: 385-391, 2017.

2. Keppel Hesselink JM: NS1209/SPD 502, A novel selective AMPA antagonist for stroke, neuropathic pain or epilepsy? drug development lessons learned. Drug Dev Res 78: 75-80, 2017.

3. Seto SW, Chang D, Jenkins A, Bensoussan A and Kiat HQ: Angiogenesis in ischemic stroke and angiogenic effects of Chinese herbal medicine. J Clin Med 7: 630-645, 2016.

4. Vasileva D, Lubenova D, Mihova M, Grigorova-Petrova K and Dimitrova A: Errata corrige. Orthostatic reactivity in patients with ischemic stroke in the chronic period. http://dx.doi. org/10.3889/oamjms.2015.090. Open Access Maced J Med Sci 3: 531-536, 2015

5. Boisserand LS, Kodama T, Papassin J, Auzely R, Moisan A, Zme C and Detante O: Biomaterial applications in cell-based therapy in experimental stroke. Stem Cells Int 68: 2577-2584, 2016.

6. Liang Y, Huang J, Tian J, Cao Y, Zhang G, Wang C, Cao Y and $\mathrm{Li} \mathrm{J}$ : The prevalence and risk factors of stroke in patients with chronic schizophrenia. Neuropsychiatr Dis Treat 9: 1131-1134, 2016.

7. Crivera C, Nelson WW, Schein JR and Witt EA: Attitudes toward anticoagulant treatment among nonvalvular atrial fibrillation patients at high risk of stroke and low risk of bleed. Patient Prefer Adherence 17: 795-805, 2016.

8. Wang Y and Bajorek B: Clinical pre-test of a computerized antithrombotic risk assessment tool for stroke prevention in atrial fibrillation patients: Giving consideration to NOACs. J Eval Clin Pract 206: 26-32, 2016

9. Hastrup S, Damgaard D, Johnsen SP and Andersen G: Prehospital acute stroke severity scale to predict large artery occlusion: Design and comparison with other scales. Stroke 47: 1772-1776, 2016
10. Zhang H, Li L, Sun Y, Zhang X, Zhang Y, Xu S, Zhao P and Liu T: Sevoflurane prevents stroke-induced depressive and anxiety behaviors by promoting cannabinoid receptor subtype I-dependent interaction between $\beta$-arrestin 2 and extracellular signal-regulated kinases $1 / 2$ in the rat hippocampus. J Neurochem 137: 713-725, 2016.

11. Oshiro AH, Otsuki DA, Hamaji MW, Rosa KT, Ida KK, Fantoni DT and Auler JO Jr: Pulse pressure variation and stroke volume variation under different inhaled concentrations of isoflurane, sevoflurane and desflurane in pigs undergoing hemorrhage. Clinics (São Paulo) 70: 577-590, 2015.

12. Gauberti M, Obiang P, Guedin P, Balossier A, Gakuba C, Diependaele AS, Chazalviel L, Vivien D, Young AR, Agin V and Orset C: Thrombotic stroke in the anesthetized monkey (Macaca mulatta): Characterization by MRI-a pilot study. Cerebrovasc Dis 33: 329-339, 2012.

13. Hinohara H, Kadoi Y, Takahashi K, Saito S, Kawauchi C and Mizutani A: Time course of changes in cerebral blood flow velocity after tourniquet deflation in patients with diabetes mellitus or previous stroke under sevoflurane anesthesia. J Anesth 25: 435-447, 2011.

14. Fang Y, Huang X, Wan Y, Tian H, Tian Y, Wang W, Zhu S and Xie M: Deficiency of TREK-1 potassium channel exacerbates secondary injury following spinal cord injury in mice. J Neurochem 141: 236-246, 2017.

15. Lu L, Zhang G, Song C, Wang X, Qian W, Wang Z, Liu Y, Gong S and Zhou S: Azrachidonic acid has protective effects on oxygen-glucose deprived astrocytes mediated through enhancement of potassium channel TREK-1 activity. Neurosci Lett 636: 241-247, 2017.

16. Tomuschat C, O'Donnell AM, Coyle D, Dreher N, Kelly D and Puri P: Altered expression of a two-pore domain (K2P) mechano-gated potassium channel TREK-1 in Hirschsprung's disease. Pediatr Res 80: 1787-1799, 2016.

17. Zhang M, Yin HJ, Wang WP, Li J and Wang XL: Over-expressed human TREK-1 inhibits $\mathrm{CHO}$ cell proliferation via inhibiting PKA and p38 MAPK pathways and subsequently inducing G1 arrest. Acta Pharmacol Sin 37: 25-32, 2016.

18. Levitz J, Royal P, Comoglio Y, Wdziekonski B, Schaub S, Clemens DM, Isacoff EY and Sandoz G: Heterodimerization within the TREK channel subfamily produces a diverse family of highly regulated potassium channels. Proc Natl Acad Sci USA 113: 266-271, 2016.

19. Fukasaku M, Kimura J and Yamaguchi O: Swelling-activated and arachidonic acid-induced currents are TREK-1 in rat bladder smooth muscle cells. Fukushima J Med Sci 62: 97-110, 2016.

20. Vivier D, Bennis K, Lesage F and Ducki S: Perspectives on the two-pore domain potassium channel TREK-1 (TWIK-related $\mathrm{K}(+)$ channel 1). A novel therapeutic target? J Med Chem 59: 328-335, 2016. 